\title{
Current management of the gastrointestinal complications of systemic sclerosis
}

Anton Emmanuel

Gastrointestinal Physiology Unit, University College Hospital, 235 Euston Road, London NW1 2BU, UK.

a.emmanuel@ucl.ac.uk

\begin{abstract}
Systemic sclerosis is a multisystem autoimmune disorder that involves the gastrointestinal tract in over $90 \%$ of patients. This involvement can extend from the mouth to the anus, with the oesophagus and anorectum being most frequently affected. Gut complications result in a plethora of presentations that impair oral intake and faecal continence and, consequently, have an adverse effect on patient quality of life, resulting in referral to gastroenterologists. The cornerstones of gastrointestinal symptom management are to optimize symptom relief and monitor for complications, in particular anaemia and malabsorption. Early intervention in patients who develop these complications is critical to minimize disease progression and improve prognosis. In the future, enhanced therapeutic strategies should be developed, based on an improved understanding of the intestinal pathophysiology of systemic sclerosis. This Review will describe the most commonly occurring clinical scenarios of gastrointestinal involvement in patients with systemic sclerosis as they present to the gastroenterologist, with recommendations for the suggested assessment protocol and therapy in each situation.
\end{abstract}

\section{Key points}

- Gut involvement in systemic sclerosis occurs more commonly in the diffuse than the limited cutaneous variant

- Oesophageal involvement is the most common gastrointestinal manifestation of systemic sclerosis, with severe reflux disease and dysphagia the main presentations 
- When treating small intestinal bacterial overgrowth in patients with systemic sclerosis, effectiveness of treatment and decisions about cyclical courses of antibiotic are best assessed by symptoms rather than breath testing

- Malnutrition in patients with systemic sclerosis is associated with more aggressive disease progression, and the risk of developing malnutrition is related to both gut and extraintestinal factors

- Faecal incontinence in systemic sclerosis is related to anal sphincter atrophy and is poorly responsive in the long-term to sacral neuromodulation

Systemic sclerosis is a chronic autoimmune-mediated connective tissue disorder. Although the aetiology of the disease remains undetermined, systemic sclerosis is characterized by fibrosis and proliferative vascular lesions of the skin and internal organs. Prevalence of the disease in Europe and the USA is estimated at 8-30 per 100,000 people, with an annual incidence of 1-2 per 100,000 people ${ }^{[1-3]}$. Incidence of systemic sclerosis is twice as frequent in black compared with white populations, and the disease is between twofold and fourfold more common in women than in men; peak age of disease onset is $30-50$ years ${ }^{[2,3]}$. Systemic sclerosis is classified as either diffuse or limited cutaneous disease, based on the extent of skin involvement, with limited systemic sclerosis accounting for $\sim 80 \%$ of diagnoses ${ }^{[4]}$. Patients with limited systemic sclerosis have skin thickening (scleroderma) only distal to the elbows and knees, but the face can be affected in those with diffuse or limited disease. This classification also defines the organ involvement, autoantibody profile and clinical progression of the disease $^{[5,6]}$ Diffuse disease is characterised by having positive ScL70, early and rapid organ involvement and with a poor prognosis. Limited systemic sclerosis is characterised by positive anti-centromere antibodies, slow progression, visceral involvement later in the disease course and a better prognosis. Involvement of the gastrointestinal tract can be found in either form, 
but is more common in patients with diffuse disease ${ }^{[6]}$. Gastrointestinal symptoms are seen in up to $90 \%$ of all patients, and are the presenting feature of disease in $10 \%$ of individuals ${ }^{[7]}$. Gastrointestinal involvement is the leading cause of morbidity and third most common cause of mortality in patients with systemic sclerosis, with cardiopulmonary and renal involvement being first and second, respectively ${ }^{[7]}$.

\section{Gastrointestinal involvement}

Vascular endothelial hyper-reactivity manifested as recurrent episodes of vasoconstriction, and reperfusion has been hypothesized to be the initial pathological event in the development of systemic sclerosis ${ }^{[8]}$. Sequential histological studies of enteric smooth muscles from patients with systemic sclerosis suggest that the initial trigger for this vascular instability is autonomic axonal degeneration, specifically sympathetic overactivity ${ }^{[9,10]}$. Alternatively, the initiating event has also been hypothesized to be autoimmune related, on the basis of immunohistochemical studies showing the presence of circulating $\operatorname{IgG}$ autoantibodies against myenteric neurons in many patients with systemic sclerosis ${ }^{[11]}$. Currently available data support a combination of the autoimmune and autonomic aetiology hypotheses, with the identification that circulating $\operatorname{IgG}$ autoantibodies are capable of disrupting intestinal myoelectrical function ${ }^{[11,12]}$. Specifically, patients with systemic sclerosis express antibodies to the muscarinic acetylcholine receptor M3, which is critical for the control of intestinal peristalsis by the enteric nervous system ${ }^{[12,13]}$. Patients with more severe gastrointestinal phenotypes have increased expression of these antibodies, supporting a causal link between antibody levels and gastrointestinal systemic sclerosis features ${ }^{[13]}$.

Whatever the aetiology of the disease, vascular instability is thought to progress to tissue ischaemia and subsequent defective immunity and tissue repair. Tissue repair in patients with 
systemic sclerosis is characterized by excessive collagen and collagen matrix deposition, resulting in fibrotic replacement of tissue in the skin and internal organs, including in the gastrointestinal tract ${ }^{[14]}$. Fibrosis involves both the gut smooth muscle and the smooth muscle of the gastrointestinal vasculature ${ }^{[15]}$. This fibrotic infiltration in the gut results in dysmotility and delayed transit time, which can affect any gastrointestinal region from the oral cavity to the anus ${ }^{[8,16,17]}$.

Gut involvement can progress slowly or rapidly ${ }^{[18,19]}$. However, several studies have failed to show any correlation between gastrointestinal symptoms and severity of physiological compromise in systemic sclerosis ${ }^{[8,10]}$. There also seems to be no correlation between patientreported severity of gut symptoms and degree of morbidity of systemic sclerosis ${ }^{[20]}$. Severe gastrointestinal involvement, especially small bowel involvement presenting with malabsorption and dysmotility, is associated with increased mortality in some series ${ }^{[4,21]}$. This finding emphasises the importance of early and continuous monitoring of gastrointestinal tract involvement in patients with systemic sclerosis to avoid these severe syndromes of small bowel failure $^{[22]}$. Optimal monitoring assessments and emerging potential therapies will be detailed in this Review, which will be structured around presentation symptoms and syndromes, rather than anatomical location, so as to have the greatest utility to the clinician seeing these patients.

\section{Dysphagia}

Although oesophageal dysmotility is the most common gastrointestinal manifestation of systemic sclerosis $^{[18,23,24]}$, it is vital to consider that dysphagia might be caused by a GERDrelated stricture (discussed later), and hence an upper gastrointestinal endoscopy might be appropriate to exclude this possibility. In turn, however, the absence of oesophageal symptoms does not necessarily exclude oesophageal involvement since physiological change may precede 
symptom development ${ }^{[20,25]}$. Whether the prevalence and severity of oesophageal dysmotility is related to the extent or severity of cutaneous systemic sclerosis involvement is unclear ${ }^{[26,27]}$. Oesophageal manometry has superseded scintigraphy and barium radiography as a diagnostic modality since it is more reproducible, more interpretable and more available. High-resolution manometry might reveal more about the pathophysiology of dysphagia in systemic sclerosis, as our understanding of this methodology improves, given its ability to demonstrate subtle topographic change not necessarily picked up by conventional manometry. The characteristic motility abnormalities in patients with systemic sclerosis are: reduced (or absent) lower oesophageal sphincter pressure; ineffective oesophageal body peristalsis (especially in the distal oesophagus); and incoordination of peristaltic and lower oesophageal sphincter function $^{[8,15,27,28,29]}$. Pharyngeal and proximal oesophageal motility is normal in systemic sclerosis ${ }^{[30]}$. This may reflect the somatic innervation of the proximal oesophagus, which is relatively spared compared to visceral innervation.

To date, no pharmacological intervention has been shown to influence the development of the oesophageal complications of systemic sclerosis and, consequently, treatment focuses on symptomatic management with prokinetic agents ${ }^{[22]}$. Prokinetic agents stimulate gut peristalsis through a direct receptor mechanism on enterocytes, as opposed to laxatives which tend to have a non-specific mucosal contact action. Considerable data exists on the acute manometric effects of prokinetics such as metoclopramide, erythromycin, bethanechol, buspirone and domperidone, and these studies have been reviewed elsewhere ${ }^{[26]}$, but no formal studies have been published on the use of these agents in clinical practice. Prucalopride might have a role as a prokinetic, given its mechanism of increasing cholinergic tone in the gut and hence enhancing peristalsis in upper and lower gastrointestinal tract ${ }^{[31,32]}$. In any event, prokinetic agents probably have no effect in advanced systemic sclerosis when smooth muscle atrophy 
would preclude their being beneficial. In terms of non-pharmacological therapies, a study of patients with early systemic sclerosis suggested a role for low-frequency electrical acupuncture stimulation to improve dysphagia ${ }^{[33]}$. In brief, the technique involves insertion of stainless steel acupuncture needles in designated points and attached to an electrical stimulator at variable frequencies, the intensity being adjusted to produce local muscle contractions without pain and discomfort. The uncontrolled study included 34 patients with systemic sclerosis and showed modest clinical benefits in tandem with modest changes in inflammatory biopsies from cutaneous biopsy pre- vs post-treatment. Treatment duration varied in studies from 3 months to 9 years. Transcutaneous electrical nerve stimulation (TENS) has not shown efficacy in patients with more advanced disease ${ }^{[34]}$. High-frequency TENS applied during oesophageal manometry showed no change in any motility parameters. There is no data on low frequency TENS or patients with early SSc

\section{GERD}

The manometric features described earlier demonstrate a strong predisposition in patients with systemic sclerosis to pathological GERD; over three-quarters of patients with systemic sclerosis have erosive reflux changes in the oesophagus ${ }^{[35]}$, and degree of erosive correlates with change and severity of dysmotility ${ }^{[36]}$. As such, monitoring of patients with systemic sclerosis by simultaneous recording of oesophageal $\mathrm{pH}$ and manometry has been recommended, regardless of the presence or absence of reflux symptoms ${ }^{[37]}$. Fifty percent of patients with systemic sclerosis have delayed gastric emptying ${ }^{[15,38,54]}$ which also predisposes to GERD. The severity and chronicity of GERD in patients with systemic sclerosis results in high rates of stricturing and the development of Barrett oesophagus in $40 \%{ }^{[39]}$. 
Conservative therapy of GERD symptoms (lifestyle modification, postural assistance and dietary modification) has not been formally studied in patients with systemic sclerosis, but given the severity of physiological disturbance in these individuals such treatments are rarely effective in isolation ${ }^{[39,40]}$. Nevertheless, eating small meals, avoiding meals shortly before recumbence, reducing fat intake and ceasing smoking are all reasonable approaches ${ }^{[26,40]}$. PPIs are the core of GERD maintenance therapy, and might need to be used in double or even quadruple doses $^{[41,42]}$. Given the increasingly well-recognized adverse effects of chronic PPI use $^{[43]}$ and the need for chronic therapy in patients with systemic sclerosis, counselling patients at initiation of treatment is important. Adverse effects relevant to patients with systemic sclerosis are the association of PPIs with osteopaenia, intestinal infection (including small intestinal bacterial overgrowth (SIBO)) and interaction with antiplatelet therapies. Based on a comparator study with the 5-hydroxytrypamine receptor 2 (also known as serotonin receptor 2) agonist cimetidine, use of antacids is not supported in systemic sclerosis, but provided that aluminium-containing agents (which can cause constipation) are avoided, they might be a lowrisk alternative. The efficacy of adding 5-hydroxytrypamine receptor 2 agonists to PPIs improve GERD symptoms has not been studied in patients with systemic sclerosis, yet benefit is minimal in patients with GERD who do not have systemic sclerosis ${ }^{[44]}$. More aggressive modalities such as endoscopic or laparoscopic antireflux procedures are also unreported in systemic sclerosis and are best avoided as they are likely to be complicated by dysphagia, given the known severe dysmotility in these patients ${ }^{[8,15]}$.

A key focus of GERD therapy is to avoid progression to more complex manifestations such as oesophageal strictures or Barrett oesophagus. Endoscopic dilatation is indicated in the $\sim 30 \%$ of patients with systemic sclerosis who develop strictures which will manifest as progressive dysphagia. ${ }^{[45]}$, in addition to optimizing PPI therapy to minimize stricture recurrence. 
Prospective studies of Barrett oesophagus in patients with systemic sclerosis showed an annual $3 \%$ rate of progression to high-grade dysplasia and $0.7 \%$ incidence of oesophageal adenocarcinoma $^{[46,47]}$. Discussion of the frequency of screening for Barrett oesophagus is beyond the scope of this Review, but the high incidence of high-grade dysplasia in patients with systemic sclerosis mandates frequent endoscopic monitoring. There is no guidance on the frequency of such monitoring, but it should be influenced by multi-disciplinary assessment of severity of symptoms, degree and rate of histological change.

An emerging important complication of GERD seems to be the role of micro-aspiration of gastric content as a driver of interstitial lung disease. Initial data in an animal model ${ }^{[48]}$ showed that gastric content introduced into the lung caused a parenchymal fibrotic reaction. This finding has been supplemented with data in patients with systemic sclerosis ${ }^{[49]}$ correlating increased degree of lung fibrosis with more frequent reflux episodes and greater proximal extent of refluxate. The relationship between reflux and development of pulmonary involvement is equivocal: $50 \%$ of patients with systemic sclerosis with severe reflux associated with lung disease risk were asymptomatic from the oesophageal perspective, and progression of lung disease was not associated with progressive hypomotility ${ }^{[50,51]}$. There is, as yet, no trial data showing improvement in the lung function of patients with systemic sclerosis after aggressive GERD management. However the prospective cohort data on progression of oesophageal motility and pulmonary pathophysiology supports the use of PPIs with add-on prokinetic agents, even in asymptomatic patients ${ }^{[50]}$. This view has been supported by the identification of a novel histological form of lung disease, known as centrilobular fibrosis which is seen especially in those patients with systemic sclerosis who also have severe GERD [51].

\section{Vomiting and abdominal pain}


Delayed gastric emptying is seen in $\sim 50 \%$ of patients with systemic sclerosis ${ }^{[52,53]}$. This functional delay seems to relate to both structural changes (reduced gastric size) ${ }^{[54]}$ and slowing of gastric electrical activity associated with the autonomic nerve [37,55]. The typical gastroparesis symptoms of abdominal bloating, pain, early satiety and vomiting are more commonly reported in diffuse rather than limited cutaneous systemic sclerosis ${ }^{[56]}$. None of these features are unique to systemic sclerosis, as they are also observed in functional dyspepsia as well as other causes of gastroparesis such as diabetes mellitus. As such, these symptoms cannot be recommended as clinically useful for diagnosis of systemic sclerosis or monitoring of the disease. This is especially disappointing because the correlation between symptom burden and objective abnormalities is poor ${ }^{[56]}$, leaving no widely accepted gold standard to measure outcome.

As with GERD management, there is no evidence specific for patients with systemic sclerosis in support of lifestyle and dietary therapies to manage delayed gastric emptying. Low-residue diets (which reduce stool frequency and liquidity by reducing fibre and gut stimulants such as dairy) and vitamin supplementation have been recommended on an empirical basis ${ }^{[8]}$. Equally disappointing is the lack of efficacy of prokinetic and antiemetic drugs in patients with systemic sclerosis. With regards to prokinetics, erythromycin (as opposed to domperidone) has been studied in this patient group ${ }^{[57]}$, but accelerates gastric emptying at a dose (200 $\mathrm{mg}$ ) while also inhibiting small bowel motility ${ }^{[58]}$. Symptom response data beyond 33 weeks for erythromycin is lacking, and the drug is most effective in early disease, before smooth muscle atrophy and fibrosis set $\mathrm{in}^{[57]}$. A consensus guideline of the management of gastroparesis in general suggested that the liquid formulation of metoclopramide can be considered if no neurological or electrocardiographic adverse effects develop, although there is no specific evidence in SScassociated gastroparesis ${ }^{[59]}$. Octreotide does not seem to have an effect on gastric emptying or 
gastroparetic symptoms $^{[60]}$. A placebo-controlled crossover study of infusion with the gastricderived prokinetic hormone ghrelin ${ }^{[61]}$ in 10 patients with systemic sclerosis showed an acceleration of gastric emptying (53 minutes saline vs 43 minutes ghrelin) ${ }^{[62]}$, raising the potential for ghrelin analogues as therapy. Jejunal feeding can be considered in situations in which there is symptomatic delay in gastric emptying in the presence of normal small bowel transit ${ }^{[63]}$ : this treatment and parenteral nutrition will be discussed in detail later. A singlecentre study of transcutaneous electrical nerve stimulation has shown short-term (two weeks) efficacy in improving gastroparesis symptoms associated with systemic sclerosis ${ }^{[64]}$. Surgical procedures such as venting gastrectomy or antrectomy are associated with major long-term complications $^{[65]}$ and should only be considered in the most extreme of situations such as intractable vomiting, anorexia and profound progressive weight loss ${ }^{[8]}$.

\section{Small intestinal bacterial overgrowth}

The clinical symptoms of SIBO are abdominal bloating, flatulence, early satiety, post-prandial nausea and, occasionally, diarrhoea ${ }^{[20,26,40]}$. Disturbances of small intestinal motility and immunity can be compounded by hypochlorhydria secondary to PPI usage and hence predispose to migration and colonization of the small intestine by colonic microbiota ${ }^{[66,67]}$. If not corrected SIBO can result in malnutrition, so treatment is essential, not just from the symptom-relief perspective. Hydrogen breath testing (after either a glucose or lactulose meal) is widely used as the clinical standard for diagnosis of SIBO, but analyses in the past few years ${ }^{[68,69]}$ have shown that the specificity and sensitivity of such testing is no better than 68 and $44 \%$ respectively. However, the intrusiveness and comparative expense of the research standard, upper gastrointestinal endoscopy with culture of duodenal aspirate ${ }^{[67]}$, makes breath testing the most widely used, and in the author's opinion the most valid, modality for diagnosis. The alternative to testing for SIBO is to treat empirically with broad-spectrum antibiotics 
(against both aerobic and anaerobic strains) to modify the microbiota sufficiently to result in symptomatic improvement, rather than attempting to eradicate the specific $\operatorname{strain}^{[70]}$.

Antibiotic therapy is as effective at improving symptoms in patients with systemic sclerosis and $\mathrm{SIBO}^{[71]}$ as it is in patients with IBS ${ }^{[69]}$. A meta-analysis ${ }^{[72]}$ of antibiotic therapy for SIBO (caused by a variety of aetiologies beyond just SSc) published in 2013 identified SIBO symptom improvement in approximately two-thirds of patients, with no statistically significant advantage of any one agent over another. Antibiotics used in the treatment of SIBO in the analysed trials include amoxicillin, ciprofloxacin (and other quinolones), doxycycline (and other tetracyclines), metronidazole, rifaximin (and other minimally absorbable antibiotics) and trimethoprim-sulfamethoxazole. The outcome of the meta-analysis was to use any of these antibiotics for 10-14 days and to monitor outcome by symptoms rather than breath testing given the poor sensitivity of the latter ${ }^{[72]}$. In the clinical context of systemic sclerosis, in which SIBO can be recurrent due to the underlying pathophysiology in the gut, some experts argue in favour of cyclical courses of antibiotic for patients with recurrent proven SIBO (by culture of endoscopically obtained duodenal aspirate) — for example, 10-14 days treatment every month, rotating between the antibiotics listed previously to protect against the development of antibiotic resistance ${ }^{[20,72,73]}$. An alternative treatment strategy in this situation is to give a probiotic rather than antibiotic when the initial course of antibiotic is withdrawn, although there is no clear evidence to recommend any specific product ${ }^{[74]}$. Probiotic treatment is supported by a small body of clinical trial data in idiopathic $\mathrm{SIBO}^{[75]}$ as well as SIBO associated with systemic sclerosis ${ }^{[76]}$, but definitive controlled trials are needed before such an approach can be unequivocally advocated.

\section{Malnutrition}


Malnutrition in patients with systemic sclerosis is usually multifactorial in origin. Oral food intake is often reduced as a result of the persistent and debilitating symptoms of nausea, vomiting and early satiety caused by gut dysmotility, and SIBO can cause maldigestion and malabsorption of specific nutrients. The basic assessments suggested by an expert USA panel comprised full blood count, haemoglobin, vitamin $\mathrm{A}$, folic acid, ferritin and vitamin $\mathrm{B}_{12}$. In addition, extraintestinal contributors to malnutrition are often present in patients with systemic sclerosis: perioral sclerosis means that patients tend to eat a low-residue diet with decreased mineral and vitamin intake due to mechanical and pain factors when trying to eat fruit and vegetables; intake of nutrients is often exacerbated by oesophageal dysmotility. In addition, finger contractures can make preparing and eating meals arduous. Finally, low mood and adverse effects of concomitant therapy (such as calcium channel antagonists, prostaglandin derivatives, immune-suppressants and opioids) can reduce appetite ${ }^{[20,77]}$.

Malnutrition develops insidiously and so the correct choice of screening modality is unclear. A study using the malnutrition universal screening tool (MUST) ${ }^{[78]}$ identified that $28 \%$ of a cohort of 586 systemic sclerosis patients in Canada were at medium or high risk of malnutrition (MUST scores of 1 and $\geq 2$, respectively) ${ }^{[79]}$. Increased risk was associated with the degree of gastrointestinal symptomatology assessed by trial-specific questionnaire, shorter disease duration and overall systemic sclerosis disease severity (gauged by physician rating) ${ }^{[79]}$. Thus, both gut and extraintestinal factors predict the risk of developing malnutrition. Given that malnutrition is associated with more-aggressive disease progression ${ }^{[73,77]}$, screening for malnutrition is therefore recommended in all patients with systemic sclerosis. Whether this association is causal remains to be determined by suitably designed prospective cohort studies. This screening should be considered with the MUST tool, a gastrointestinal symptom assessment (using a questionnaire such as the University of California, Los Angeles 
Scleroderma Clinical Trial Consortium Gastrointestinal Tract (UCLA SCTC GIT) 2.0 instrument, discussed in detail later ${ }^{[80]}$ ) and serology comprising full blood count, haematinics, and levels of serum carotene and prealbumin ${ }^{[40,74,77]}$. Such screening is supported by the observation that nutritional treatment improves nutritional status ${ }^{[81]}$ and, hopefully, in turn will improve survival; however, prospective long-term studies are required to confirm this hypothesis.

The key to managing the patients with malnourishment related to systemic sclerosis is to have a framework for practice, underpinned by close multidisciplinary cooperation between gastrointestinal and rheumatological teams ${ }^{[74,77]}$. In the author's opinion, if present, SIBO should be treated first. In addition, gastrointestinal radiology to exclude chronic intestinal pseudo-obstruction (CIPO, discussed in more detail later) is recommended; if there is gut dilatation suggestive of CIPO then a trial of prokinetic therapy is appropriate.

If the patient remains malnourished or symptomatic despite these medical therapies then they might need to be considered for enteral nutrition ${ }^{[77]}$, either by temporary transnasal or more permanent endoscopic tube insertion. On the basis that gastroparesis can be severe in patients with systemic sclerosis, the enteral feed is preferably jejunal rather than gastric ${ }^{[22,77]}$. If enteral feeding is not practicable or successful and malnutrition continues, consideration of parenteral feeding is appropriate ${ }^{[82]}$. Despite the well-recognized complications of this approach, which include deterioration of liver function, catheter sepsis and venous thrombosis, the benefits in terms of weight gain and quality of life of home parenteral feeding have been established in patients with systemic sclerosis ${ }^{[82]}$. Additionally, patients with systemic sclerosis are not at higher risk of these complications than patients with intestinal failure secondary to other causes $^{[22,82]}$. 


\section{Chronic intestinal pseudo-obstruction}

CIPO is characterized by clinical and radiological evidence of chronic episodic intestinal obstruction in the absence of a mechanical lesion ${ }^{[83]}$; it occurs as a result of a visceral myopathy or neuropathy and can be primary or, more commonly, secondary to diseases such as systemic sclerosis $^{[84]}$. As there is no mechanical lesion, a critical goal of treatment is to avoid surgery, which by definition cannot be curative ${ }^{[83]}$. Although a wealth of data exists about the natural history and poor prognosis of CIPO in general ${ }^{[83,84,85]}$, only one case-control series on patients with systemic sclerosis hospitalized with CIPO has been published ${ }^{[86]}$. Of 64 cases, a high proportion (9\%) underwent surgical resection and an even larger proportion (16\%) died. Onequarter of the 64 patients were given home parenteral nutrition. Mortality in this case-control series was associated with malnutrition at admission (reinforcing the importance of screening and treating this gastrointestinal complication of systemic sclerosis) and was higher in men than in women ${ }^{[86]}$.

First-line therapy for acute CIPO is to rehydrate the patient, establish analgesia (using paracetamol and non-steroidals, preferably avoiding opioids that tend to exacerbate intestinal dysmotility) and decompress the bowel by nasogastric intubation. Addressing nutritional needs early on is also important while waiting for CIPO symptoms to abate. Gut prokinetic agents can help in this regard, whether or not they are being used in the chronic setting ${ }^{[87,88]}$. As discussed earlier, metoclopramide and domperidone are often of little value. Erythromycin at doses of $1.5-2 \mathrm{~g}$ per day can be of benefit in some patients with CIPO, including when CIPO is secondary to systemic sclerosis ${ }^{[87]}$. Intravenous neostigmine might help abort an episode of pseudo-obstruction, but cardiac and cholinomimetic adverse effects might preclude use in patients who are frail ${ }^{[89]}$. Subcutaneous octreotide, a somatostatin analogue, has proved 
beneficial in several series of patients with systemic sclerosis with CIPO in terms of improving gut motility and alleviating symptoms ${ }^{[90,91,92]}$. Dosing commences at $50 \mu$ g twice a day and can be increased to a maximum of $200 \mu \mathrm{g}$ per day according to treatment response ${ }^{[90,91]}$. A combination of oral erythromycin and subcutaneous octreotide has been suggested ${ }^{[58]}$, but as the latter seems to delay gastric emptying caution might be needed in patients with systemic sclerosis and a combination of CIPO and gastroparesis ${ }^{[58]}$. A newer long-acting octreotide preparation could be a better tolerated alternative to the combination of erythromycin and daily subcutaneous octreotide for prevention of relapse ${ }^{[92]}$. Finally, prucalopride has been shown to reduce the frequency of relapse of patients with CIPO, including those with CIPO related to systemic sclerosis, in a 1-year placebo-controlled study ${ }^{[88]}$.

\section{Anaemia}

Anaemia in systemic sclerosis is often the anaemia of chronic illness ${ }^{[93]}$, and can be exacerbated by the presence of major systemic sclerosis complications such as renal impairment and pulmonary hypertension ${ }^{[94]}$. Anaemia can also occur as part of the malnutrition associated with SIBO and systemic sclerosis ${ }^{[77]}$. Gastric antral vascular ectasia (GAVE) is a rare complication of systemic sclerosis (affecting $1-5 \%$ of patients with systemic sclerosis ${ }^{[95,96]}$ ) that can cause anaemia with or without overt gastrointestinal bleeding. Although GAVE has endoscopically characteristic appearances ('watermelon stomach' created by stripes of ectatic vasculature amongst the oedematous gastric mucosa, the condition is defined histologically by mucosal capillary dilatation with fibrin thrombi, foveolar epithelial changes and fibromuscular hyperplasia ${ }^{[97]}$. GAVE is most prevalent in early diffuse cutaneous systemic sclerosis, characterised by rapid skin progression, and a distinct antibody profile (anti-RNA polymerase III positivity) ${ }^{[97]}$. 
Management of GAVE in systemic sclerosis is conservative in the first instance, with iron supplementation and monitoring of haemoglobin levels to minimize the duration of therapy, which can exacerbate constipation ${ }^{[96]}$. Endoscopic therapy with a neodymium-doped ytrrium aluminium garnet (Nd:YAG) laser or argon plasma coagulation is indicated if oral iron supplementation fails ${ }^{[98]}$. Efficacy is similar with both endoscopic treatments (about 90\%) but multiple treatment sessions are often required with either modality ${ }^{[99,100,101]}$. However, argon plasma coagulation is generally preferred due to its lower cost and reduced risk of complications, specifically haemorrhage and need for surgery ${ }^{[101]}$.

In refractory cases in which expert endoscopy is available, evidence from individual series support the use of endoscopic band ligation ${ }^{[102]}$, sclerotherapy ${ }^{[103]}$ and cryotherapy ${ }^{[104]}$ for the management of GAVE associated with systemic sclerosis. In cases refractory to these endoscopy techniques intravenous cyclophosphamide has been used in a series of three patients ${ }^{[105]}$ as well as surgical antrectomy ${ }^{[106]}$. Whilst effective, these therapies are associated with morbidity and a mortality of approximately $7 \%$. In a randomized trial, high-dose chemotherapy followed by autologous haematopoietic stem cell transplantation (HSCT) has been shown to be superior to cyclophosphamide in improving skin and lung function as well as quality of life in patients with systemic sclerosis ${ }^{[107]}$, and a report published in 2015 showing a benefit for HSCT in patients with systemic sclerosis and GAVE raises the possibility of curative therapy for this gastrointestinal complication ${ }^{[108]}$

\section{Constipation}

Altered colonic physiology is seen in between $20-50 \%$ of patients with systemic sclerosis, and unlike upper gut involvement this condition is often asymptomatic ${ }^{[18,26,77]}$. Progressive colonic smooth muscle atrophy results in diminished or absent peristalsis ${ }^{[109]}$, markedly delayed 
colonic transit ${ }^{[110]}$ and, rarely, colonic pseudo-obstruction ${ }^{[91]}$. Occasionally systemic sclerosis can result in loss of colonic compliance and even stricturing ${ }^{[111,112]}$. In view of these impairments of colonic physiology, and the possibility of common coincidental colonic disease (such as diverticulosis and neoplasia), structurally assessing the colon (endoscopy or radiology) in a new presentation of constipation is important in patients with systemic sclerosis.

Because slow transit is a frequent accompaniment to systemic-sclerosis-associated constipation, a high dietary fibre intake is likely to be poorly effective and is associated with frequent bloating and flatulence ${ }^{[113,114]}$. Optimizing liquid intake can be difficult, especially if the patient has renal involvement. Similarly, stimulant laxatives (such as senna or bisacodyl) are preferred over macrogol osmotic laxatives in this situation since they cause electrolyte loss ${ }^{[114]}$. Lactulose can be effective, but only usually for 1-3 months since bacterial catabolism of the drug develops; the drug is also associated with poor tolerability and the need for dose escalation $^{[40,113]}$. In uncontrolled single-centre series, a variety of prokinetic drugs have been shown to improve colonic motility (and to a lesser extent symptoms) in systemic sclerosis: metoclopramide $20-30 \mathrm{mg}$ per day ${ }^{[109]}$, domperidone (maximum daily dose currently is $30 \mathrm{mg}$ per day in divided doses $)^{[8]}$ and prucalopride once a day $(2 \mathrm{mg}$ in all patients except over 65 year olds, in whom the dose is reduced to $1 \mathrm{mg})^{[32]}$ have been shown to improve colonic motility, whereas erythromycin ${ }^{[15]}$ and octreotide ${ }^{[8]}$ seemingly have efficacy only on small intestinal motility. If successful, long-term use of prokinetic agents is a reasonable option since the drugs seem well tolerated I the long-term with no evidence of tachyphylaxis ${ }^{[113,114]}$. Surgical resection (with or without stoma formation) is a poor option for patients with systemic sclerosis given the potential for skin complications (poor wound healing, stoma prolapse or retraction) and prolonged ileus, but might be required in extreme cases with perforation or those who are refractory to drugs ${ }^{[16,117]}$. In those patients with systemic sclerosis and 
constipation refractive to drugs, less radical options such as colonic pacing (in which an electrical impuls generator is implanted to stimulate the right colon) ${ }^{[18]}$ or sacral nerve stimulation $^{[119]}$ are appealing, but have not been studied specifically in patients with systemic sclerosis.

Air in the bowel wall (pneumatosis cystoides intestinalis) is a rare manifestation of systemic sclerosis in the colon ${ }^{[120]}$. This condition is usually inconsequential with no manifestation of symptoms, and is generally picked up by chance during abdominal radiology. On very rare occasions, a pneumoperitoneum might occur if there is dissection or rupture of these subserosal cysts.

\section{Diarrhoea}

Diarrhoea in a patient with systemic sclerosis might indicate alterations in upper gut function, not just colonic involvement. SIBO is an important consideration and evidence suggests that this condition can be identified in a patient with diarrhoea by the presence of raised faecal calprotectin levels ${ }^{[121]}$. Additional aspects of small bowel involvement can also contribute to a steatorrhoea-like presentation in systemic sclerosis ${ }^{[37,73,77,122]}$, including: intestinal ischaemia secondary to mesenteric vasculopathy; impaired lymphatic drainage secondary to fibrosis; loss of small intestinal surface area and function secondary to fibrotic infiltration; bile acid malabsorption independent of SIBO; fructose intolerance; and amyloidosis as a consequence of chronic inflammation.

For patients with systemic sclerosis and diarrhoea, the primary goal is to treat the underlying causes of intestinal dysfunction as listed previously, with consideration for the use of antibiotics for SIBO and bile acid sequestrants for bile acid malabsorption ${ }^{[122]}$. If all of these 
causes of intestinal dysfunction have been excluded or treated and diarrhoea continues, using judiciously titrated low doses of loperamide is reasonable, but caution should be exercised to avoid precipitation of severe constipation and possibly even a bout of CIPO symptoms ${ }^{[22,114]}$. A diet low in FODMAPs (Fermentable Oligosaccharides, Disaccharides, Monosaccharides And Polyols), which reduces the osmotic and fermentable substrate in the gut, is useful in improving diarrhoea in idiopathic and organic disease ${ }^{[123]}$, and could have a role in treating diarrhoea in patients with systemic sclerosis although this indication has never been studied.

\section{Faecal incontinence}

As many as $20 \%$ of patients with systemic sclerosis report faecal incontinence ${ }^{[124]}$, which leads to major effects on quality of life ${ }^{[125,126]}$. The underlying pathology is atrophy and fibrosis of the smooth muscle of the internal anal sphincter ${ }^{[127]}$ with excess distensibility of the anal canal $^{[128]}$. The most common presentation with sphincter involvement is passive faecal incontinence or soiling ${ }^{[129]}$. The alternative presentation of anorectal involvement in systemic sclerosis is rectal prolapse $\mathrm{e}^{[22,77,130]}$, which can in turn exacerbate faecal soiling as the sphincter is held open by the prolapse. Rectal prolapse in patients with systemic sclerosis results from accumulation of collagen in the rectum, which causes reduced rectal compliance and subsequent straining ${ }^{[131]}$. Anorectal involvement is characterized by reduced resting anal tone, reduced rectal balloon distensibility and an attenuated rectoanal inhibitory reflex ${ }^{[129,130,132]}$.

When stools are loose (Bristol type 6 or 7 ) augmenting fibre intake can improve symptoms ${ }^{[129]}$, yet otherwise this dietary modification is of little benefit and can even cause abdominal bloating. Antidiarrhoeals, specifically loperamide, might improve diarrhoea, but need to be used carefully to avoid exacerbating constipation and straining, which in turn is associated with increased risk of prolapse ${ }^{[22,133]}$. Suppositories can be used to optimize rectal evacuation 
without straining in this situation ${ }^{[22,113]}$. Pelvic floor biofeedback has never been studied in patients with systemic sclerosis but does warrant consideration in this population, given the nature of anorectal involvement and the known potential for biofeedback therapies in treating Raynaud phenomenon in systemic sclerosis ${ }^{[134]}$.

Sacral nerve stimulation has been studied extensively in anorectal dysfunction not related to systemic sclerosis and in patients with systemic sclerosis and faecal incontinence in a small $(n=5)$ single-centre study with 24 month follow-up ${ }^{[135]}$. However, a multisite report in a larger patient cohort has suggested that although there are no safety concerns associated with the technique, the overwhelming majority of patients with systemic sclerosis derive no sustained benefit to anorectal function from continued sacral neuromodulation ${ }^{[136]}$. A randomized placebo-controlled study published in 2014 has suggested that posterior tibial nerve stimulation could be an alternative effective method of neuromodulation and relief of faecal incontinence symptoms in systemic sclerosis ${ }^{[137]}$. Long-term follow-up of patients treated successfully in this study is needed to determine whether symptom relief is maintained.

Given potential tissue healing problems, surgery is best avoided in patients with systemic sclerosis and anorectal dysfunction. However, if there is a severe rectal prolapse, resection of the rectum and sigmoid colon is the preferred surgical approach to transanal mucosal procedures which have a high failure rate related to breakdown of the suture line ${ }^{[131]}$.

\section{Symptom questionnaires}

Gastrointestinal involvement in patients with systemic sclerosis is common and protean in manifestation. The emphasis of effective management is on early recognition, accurate categorization of regional involvement and prompt treatment to prevent the development of 
severe acute or chronic complications. In particular, avoiding malnutrition is important given its association with a worse prognosis. To that end, the central role of a validated and reproducible questionnaire for patients with systemic sclerosis has been recognized. Khanna and colleagues have pioneered the development of the UCLA SCTC GIT 2.0 instrument to address this requirement ${ }^{[80]}$. This questionnaire is an update of an earlier version, and comprises seven subscales: reflux, distention/bloating, diarrhoea, fecal soilage, constipation, emotional well-being and social functioning and a total GI score. It has been validated by other groups internationally in both limited and diffuse SSc patients, and against measures of intestinal motility ${ }^{[20,138]}$ (oesophageal manometry and anorectal physiology). In addition, the UCLA SCTC GIT 2.0 instrument also offers a potential standard against which research studies can quantify gastrointestinal symptom burden in individuals with systemic sclerosis. The instrument has been used in this way to quantify disease in patients with upper and lower gut symptoms $^{[127,138]}$. Allied to a nutrition tool such as $\operatorname{MUST}^{[78]}$, the UCLA SCTC GIT 2.0 is important in the symptom assessment of the patient with systemic sclerosis, including patients with and without gastrointestinal symptoms. In a complex multisystem disorder such as systemic sclerosis, a disease-specific questionnaire is probably of greater value than more generic questionnaires ${ }^{[139]}$, even rigorously developed patient-reported instruments such as the Patient-Reported Outcomes Measurement Information System (PROMIS) gastrointestinal symptom scale ${ }^{[140]}$. PROMIS has been shown to have construct validity in patients with systemic sclerosis, and is increasingly used in idiopathic gastrointestinal disorders including inflammatory bowel disease and irritable bowel syndrome ${ }^{[141]}$.

\section{Summary of disease management}

In patients with systemic sclerosis the gastrointestinal tract is the most commonly affected visceral system. Although oesophageal complications have received most recognition and 
study, any region of the gut can be involved. What is clear is that quality of life is reduced in patients with systemic sclerosis who develop gastrointestinal complications.

Table 1 summarizes the diagnostic considerations according to symptom presentation when managing patients with systemic sclerosis. Owing to the high prevalence of severe reflux symptoms in patients with systemic sclerosis, pragmatic, evidence-based patient management begins with the use of PPIs to control reflux symptoms and potentially reverse oesophageal and pulmonary complications. The second most commonly involved gastrointestinal site in systemic sclerosis is the anorectum, and patients should be actively screened for symptoms of constipation, diarrhoea and faecal incontinence. Laxative therapy tailored to individual needs and combined with toileting and lifestyle advice can successfully treat many patients with constipation and minimize the risk of rectal prolapse. Diarrhoea has a variety of treatable causes that can be identified with fairly simple investigation. Faecal incontinence is a disabling symptom of anorectal involvement in systemic sclerosis and might be amenable to neuromodulation therapy. As malnutrition is associated with increased disease severity and a poorer prognosis, active screening should be used to identify patients with this symptom and to target them for early intervention; screening can be accomplished by simple anthropometric measurements (such as BMI, circumferences of mid upper-arm and waist, triceps skin-fold thickness) but also by serial observation of the patient and the simple tool of the MUST. The key to successful management of this wide array of physically and socially disabling symptoms is early identification and symptom control because at the current time no therapy can reverse the pathophysiology of systemic sclerosis involvement in the digestive tract. Given the wide range of manifestations of systemic sclerosis, there is a preeminent role of the multidisciplinary team (MDT) in these patients: key members of the team would include a gastroenterologist, rheumatologist, dietitian and radiologist. 


\section{Future research directions}

Given the prevalence of gastrointestinal symptoms in systemic sclerosis, a number of avenues for future research exist. Current questionnaires should expand beyond assessment of the frequency of symptoms to also include symptom severity and the effects on patient quality of life. The expansion of these tools would require validation of the new questionnaires in patients with limited and diffuse systemic sclerosis. The potential utility of gastrointestinal diagnostics (faecal calprotectin levels, selenium homocholic acid taurine (SeHCAT) testing and hydrogen breath testing) to identify occult inflammation, bile acid malabsorption and sugar intolerances needs exploration in symptomatic and asymptomatic patients with systemic sclerosis. In addition, clinical trials of novel gastrointestinal agents such as prokinetic agents (for example, prucalopride) or secretagogues (for example, linaclotide and lubiprostone) are required to ascertain whether these gut mucosa targeted agents, which act on specific mucosal receptors have a particular efficacy in those patient groups. If the results of such trials are positive, these therapeutic agents might prevent the progression of gastrointestinal dysfunction to intestinal failure.

\section{Conclusions}

Gastrointestinal involvement is the commonest cause of morbidity and third most common cause of mortality in patients with systemic sclerosis, affecting especially those patients with diffuse rather than limited disease. Oesophageal involvement in the form of severe reflux and dysphagia are very common and need treatment to alleviate symptoms and potentially reduce pulmonary co-morbidity. Malnutrition needs to be surveyed for and managed actively. Constipation and faecal incontinence may coexist; novel drug and neurostimulation 
treatments may offer therapeutic potential. When present diarrhoea may have a variety of causes which should be examined for in order to tailor treatment. 
1. Allcock R.J. et al. A study of the prevalence of systemic sclerosis in northeast england. Rheumatology (Oxford). 43. 596-602 (2004)

2. Chifflot $\mathrm{H}$. et al. Incidence and prevalence of systemic sclerosis: a systematic literature review. Semin Arthritis Rheum. 37. 223-35 (2008)

3. Andréasson K. et al Prevalence and incidence of systemic sclerosis in southern Sweden: population-based data with case ascertainment using the 1980 ARA criteria and the proposed ACR-EULAR classification criteria. Ann Rheum Dis. 73.1788-92 (2014)

4. Steen V.D. and Medsger T.A. Jr. Severe organ involvement in systemic sclerosis with diffuse scleroderma. Arthritis Rheum. 43. 2437-44 (2000)

5. Lock G., Holstege A., Lang B. \& Scholmerich J. Gastrointestinal manifestations of progressive systemic sclerosis. Am J Gastroenterol. 92. 763-71 (1997)

6. Attar A. Digestive manifestations in systemic sclerosis. Ann Med Interne (Paris). 153. 260-4 (2002)

7. Hunzelmann N. et al The registry of the German Network for Systemic Scleroderma: frequency of disease subsets and patterns of organ involvement. Rheumatology (Oxford). 47.1185-1192 (2008)

8. Sjogren R.W. Gastrointestinal features of scleroderma. Curr Opin Rheumatol. 8. 56975 (1996)

9. Malandrini A. et al. Autonomic nervous system and smooth muscle cell involvement in systemic sclerosis: ultrastructural study of 3 cases. J Rheumatol. 27. 1203-6 (2000)

10. Dessein P.H. et al. Autonomic dysfunction in systemic sclerosis: sympathetic overactivity and instability. Am J Med. 93. 143-50 (1992)

11. Eaker E.Y. et al. Myenteric neuronal antibodies in scleroderma - passive transfer evokes alterations in intestinal myoelectric activity in a rat model. J Lab Clin Med.133: 551-6 (1999)

12. Kawaguchi Y. et al. Muscarinic-3 acetylcholine receptor autoantibody in patients with systemic sclerosis: contribution to severe gastrointestinal tract dysmotility. Ann Rheum Dis. 68. 710-714 (2009)

13. Singh J. et al. Immunoglobulins from scleroderma patients inhibit the muscarinic receptor activation in internal anal sphincter smooth muscle cells. Am J Physiol Gastrointest Liver Physiol. 297. 206-213 (2009)

14. Hendel L., Kobayasi T., Petri M. Ultrastructure of the small intestinal mucosa in progressive systemic sclerosis (PSS). Acta Pathol Microbiol Immunol Scand. 95. 41-6 (1987)

15. Ponge T, Bruley des Varannes S. Digestive involvement of scleroderma. Rev Pract. 52. 1896-900 (2002)

16. Poirier T.J. and Rankin G.B. Gastrointestinal manifestations of progressive systemic scleroderma based on a review of 364 cases. Am J Gastroenterol. 58. 30-44 (1972)

17. Young M.A., Rose S. and Reynolds J.C. Gastrointestinal manifestations of scleroderma. Rheum Dis Clin North Am. 22. 797-823 (1996)

18. Sallam P.J. et al. Assessment of gastrointestinal involvement. Clin Exp Rheumatol. 21 (3 Suppl. 29). S15-8 (2003)

19. Weber P. et al. 24-h intraesophageal $\mathrm{pH}$ monitoring in children and adolescents with scleroderma and mixed connective tissue disease. J Rheumatol. 27. 2692-5 (2000)

20. Thoua N. et al. Assessment of gastrointestinal symptoms in patients with systemic slcerosis in a UK tertiary referral centre. Rheumatol (Oxford). 49. 1770-1775 (2010)

21. Harrison E., Herrick A.L., McLaughlin J.T. and Lal S. Malnutrition in systemic sclerosis. Rheumatology. 51.1747-56 (2012) 
22. Hansi N. et al. Consensus best practice pathway of the UK scleroderma study group: gastrointestinal manifestations of systemic sclerosis. Clin Exp Rheumatol. 32(6 Suppl 86). S-214-21 (2014)

23. Marshall J.B. et al. Gastrointestinal manifestations of mixed connective tissue disease. Gastroenterology; 98: 1232-8 (1990)

24. Bestetti A. et al. Esophageal scintigraphy with a semisolid meal to evaluate esophageal dysmotility in systemic sclerosis and Raynaud's phenomenon. $\mathrm{J} \mathrm{Nucl}$ Med. 40. 77-84 (1999)

25. Akesson A. and Wollheim F.A. Organ manifestations in 100 patients with progressive systemic sclerosis: a comparison between the CREST syndrome and diffuse scleroderma. Br J Rheumatol. 28. 281-86 (1989)

26. Sallam H., McNearney T.A., Chen J.D.Z. Systematic review: pathophysiology and management of gastrointestinal dysmotility in systemic sclerosis (scleroderma). Aliment Pharmacol Ther. 23. 691-712 (2006)

27. Lepri $\mathrm{G}$ et al. Evidence for oesophageal and anorectal involvement in very early systemic sclerosis (VEDOSS): report from a single VEDOSS/EUSTAR centre. Ann Rheum Dis. 74.124-128 (2015)

28. Weston S., Thumshirn M., Wiste J. \& Carnilleri M. Clinical and upper gastrointestinal motility features in systemic sclerosis and related disorders. Am J Gastroenterol. 93. 1085-9 (1998)

29. Roman S. et al. Esophageal dysmotility associated with systemic sclerosis: a highresolution manometry study. Dis Esophagus. 24. 299-304 (2011)

30. Dantas R.O. and Aprile L. Esophageal striated muscle contractions in patients with gastroesophageal reflux symptoms. Dig Dis Sci. 47. 2586-90 (2002)

31. Emmanuel A., Kamm M.A., Roy A.J., Antonelli, K. Effect of a novel prokinetic drug, R093877, on gastrointestinal transit in healthy volunteers. Gut. 42. 511-516 (1998)

32. Boeckxstaens G.E., Bartelsman J.F., Lauwers L. \& Tytgat G.N. Treatment of GI dysmotility in scleroderma with the new enterokinetic agent prucalopride. Am J Gastroenterol. 97. 194-7 (2002)

33. Maeda M, Ichiki Y, Sumi A, Mori S. A trial of acupuncture for progressive systemic sclerosis. J Dermatol. 15. 133-40 (1988)

34. Mearin F. et al. Effect of transcutaneous nerve stimulation on esophageal motility in patients with achalasia and scleroderma. Scand J Gastroenterol. 25. 1018-23 (1990)

35. Thonhofer R., Siegel C., Trummer M., Graninger W. Early endoscopy in systemic sclerosis without gastrointestinal symptoms. Rheumatol Int. 32. 165-8 (2012)

36. Zamost B.J. et al. Esophagitis in scleroderma. Prevalence and risk factors. Gastroenterology. 92: 421-8 (1987)

37. Marie I., et al. Gastric involvement in systemic sclerosis - a prospective study. Am J Gastroenterol; 96: 77-83 (2001)

38. Katzka D.A. et al. Barrett's metaplasia and adenocarcinoma of the esophagus in scleroderma. Am J Med. 82. 46-52 (1987)

39. McMahan ZH, Hummers LK. Systemic sclerosis - challenges for clinical practice. Nat Rev Rheumatol. 2013;9:90-100.

40. Nagaraja V., McMahan Z.H., Getzug T. \& Khanna D. Management of Gastrointestinal Involvement in Scleroderma. Current Treatment Options in Rheumatology. 1. 82-105 (2015)

41. Shoenut J.P., Wieler JA. \& Micflikier AB. The extent and pattern of gastrooesophageal reflux in patients with scleroderma oesophagus: the effect of low-dose omeprazole. Aliment Pharmacol Ther. 7. 509-13 (1993) 
42. Hendel L., Hage E., Hendel J. \& Stentoft P. Omeprazole in the long-term treatment of severe gastro-oesophageal reflux disease in patients with systemic sclerosis. Aliment Pharmacol Ther. 6. 565-77 (1992)

43. Corleto V.D., Festa S., Di Giulio E. and Annibale B. Proton pump inhibitor therapy and potential long-term harm. Curr Opin Endocrinol Diab Obes. 21. 3-8 (2014)

44. Wang Y., Pan T., Wang Q., Guo Z. Additional bedtime H2-receptor antagonist for the control of nocturnal management of gastric acid breakthrough. Cochrane Database Syst Rev. 4. CD004275 (2009)

45. Zuccaro G Jr. Esophagoscopy and endoscopic esophageal ultrasound in the assessment of esophageal function. Semin Thorac Cardiovasc Surg. 13. 226-33 (2001)

46. Wipff J. et al. Outcomes of Barrett's oesophagus related to systemic sclerosis: a 3year EULAR Scleroderma Trials and Research prospective follow-up study. Rheumatology. 50. 1440-4 (2011)

47. Conio M. et al. Long-term endoscopic surveillance of patients with Barrett's esophagus. Incidence of dysplasia and adenocarcinoma: a prospective study. Am. $J$. Gastroenterol. 98. 1931-9 (2003)

48. Appel 3rd J.Z. et al. Characterization of the innate immune response to chronic aspiration in a novel rodent model. Respir Res. 8. 87-90 (2007)

49. Savarino E. et al. Gastroesophageal reflux and pulmonary fibrosis in scleroderma. A study using pH-impedance monitoring. Am J Respir Crit Care Med. 179.408-413 (2009)

50. Gilson M. et al. Prognostic factors for lung function in systemic sclerosis: prospective study of 105 cases. Eur Respir J. 35.112-7 (2010)

51. Christmann R.B. et al. Gastroesophageal reflux incites interstitial lung disease in systemic sclerosis: clinical, radiologic, histopathologic, and treatment evidence. Semin Arthritis Rheum. 40. 241-249 (2010)

52. de Souza R.B.C. et al.: Centrilobular fibrosis: an underrecognized pattern in systemic sclerosis. Respiration. 77: 389-397 (2009)

53. Madsen J.L., Hendel L. Gastrointestinal transit times of radiolabeled meal in progressive systemic sclerosis. Dig Dis Sci. 37. 1404-8 (1992)

54. Domsic R., Fasanella K., Bielefeldt K. Gastrointestinal manifestations of systemic sclerosis. Dig Dis Sci. 53. 1163-74 (2008)

55. Cozzi F. et al. Gastric dysmotility after liquid bolus ingestion in systemic sclerosis: an ultrasonographic study. Rheumatol Int. 32. 1219-23 (2012)

56. Franck-Larsson K., Hedenstrom H., Dahl R. \& Ronnblom A. Delayed gastric emptying in patients with diffuse versus limited systemic sclerosis, unrelated to gastrointestinal symptoms and myoelectric gastric activity. Scand J Rheumatol. 32. 348-55 (2003)

57. Fiorucci S., Distrufti V., Gerli R. \& Morelli A. Effect of erythromycin on gastric and gallbladder emptying and gastrointestinal symptoms in scleroderma patients is maintained medium term. Am J Gastroenterol. 89. 550-5 (1994)

58. Verne G.N., Eaker E.Y., Hardy E. \& Sninsky C.A. Effect of octreotide and erythromycin on idiopathic and scleroderma-associated intestinal pseudoobstruction Dig Dis Sci. 40. 1892-901 (1995)

59. Camilleri M. et al. American College of Gastroenterology Clinical guideline: management of gastroparesis. Am J Gastroenterol. 108. 18-37 (2013)

60. Edmunds M.C. et al. Effect of octreotide on gastric and small bowel motility in patients with gastroparesis. Aliment Pharmacol Ther. 12. 167-74 (1998) 
61. Murray C., Kamm M., Bloom S. \& Emmanuel A. Ghrelin for the gastroenterologist: history and potential. Gastroenterology. 125. 1492-1502 (2003)

62. Ariyasu H. et al. Clinical effects of ghrelin on gastrointestinal involvement in patients with systemic sclerosis. Endocr J. 61. 735-42 (2014)

63. McCallum R. et al. Clinical response to gastric electrical stimulation in patients with postsurgical gastroparesis. Clin Gastroenterol Hepatol. 3. 49-54 (2005)

64. Sallam H., Doshi D., McNearney T. \& Chen J.D.Z. Transcutaneous electrical nerve stimulation improves gastric motility, dyspeptic symptoms and physical functioning in patients with systemic sclerosis by balancing the sympathovagal activity. Gastroenterology. 128. A-468 (2005)

65. Oiwa $\mathrm{H}$. et al. A case of systemic sclerosis sine scleroderma associated with perforation of an afferent loop after subtotal gastrectomy with Bilroth 2 anastomosis for its severe gastrointestinal involvement. Modern Rheumatol / Japan Rheum Assoc. 15. 371-3 (2005)

66. You C.H. et al. Gastric and small intestinal myoelectrical dysrhythmia associated with chronic intractable nausea and vomiting. Ann Int Med. 95. 449-51 (1981)

67. Ghoshal U.C., Srivastava D., Ghoshal U. \& Misra A. Breath tests in the diagnosis of small intestinal bacterial overgrowth in patients with irritable bowel syndrome in comparison with quantitative upper gut aspirate culture. Eur J Gastroenterol Hepatol. 26. 753-60 (2014)

68. Simrén M and Stotzer P.O. Use and abuse of hydrogen breath tests. Gut. 55. 297-303 (2006)

69. Quigley E.M. Small intestinal bacterial overgrowth: what it is and what it is not. Curr Opin Gastroenterol. 30. 141-6 (2014)

70. Quigley E.M. and Quera R. Small intestinal bacterial overgrowth: roles of antibiotics, prebiotics, and probiotics. Gastroenterology. 130(2 Suppl 1). S78-90 (2006)

71. Parodi A. et al. Small intestinal bacterial overgrowth in patients suffering from scleroderma: clinical effectiveness of its eradication. Am J Gastroenterol. 103. 125762 (2008)

72. Shah S.C., Day L.W., Somsouk M. \& Sewell J.L. Metaanalysis: antibiotic therapy for small intestinal bacterial overgrowth. Aliment Pharmacol Ther. 38. 925-34 (2013)

73. Gyger G. and Baron M. Gastrointestinal Manifestations of Scleroderma: Recent Progress in Evaluation, Pathogenesis and Management. Curr Rheumatol Rep. 14. 22 29 (2012)

74. Baron M. et al. Screening and management for malnutrition and related gastrointestinal disorders in systemic sclerosis: recommendations of a North American expert panel. Clin Exp Rheumatol. 28 (Suppl. 58). 42-46 (2010)

75. Soifer L.O., Peralta D., Dima G. \& Besasso H. Comparative clinical efficacy of a probiotic vs. an antibiotic in the treatment of patients with intestinal bacterial overgrowth and chronic abdominal functional distension: a pilot study. Acta Gastroenterol Latinoam. 40. 323-7 (2010)

76. Frech T.M. et al. Probiotics for the treatment of systemic sclerosis-associated gastrointestinal bloating/distention. Clin Exp Rheumatol. 29(2 Suppl 65). S22-5 (2011)

77. Forbes A., Marie I. Gastrointestinal complications: the most frequent internal complications of systemic sclerosis. Rheumatology. 48: 36-9 (2009)

78. da Silva Fink J., Daniel de Mello P., Daniel de Mello E. Subjective global assessment of nutritional status - A systematic review of the literature. Clin Nutr. 34. 785-92 (2015) 
79. Baron M. et al. Malnutrition is common in systemic sclerosis: results from the Canadian scleroderma research group database. J Rheumatol. 36. 2737-43 (2009)

80. Khanna D. et al. Reliability and validity of the University of California, Los Angeles Scleroderma Clinical Trial Consortium Gastrointestinal Tract Instrument. Arthritis Rheum. 61. 1257-63 (2009)

81. Krause L. et al. Nutritional status as marker for disease activity and severity predicting mortality in patients with systemic sclerosis. Ann Rheum Dis. 69: 19511957 (2010)

82. Brown M. et al. Home parenteral nutrition - an effective and safe long-term therapy for systemic sclerosisrelated intestinal failure. Rheumatology (Oxford). 47. 176-9 (2008)

83. Mann S.D., Debinski H.S. \& Kamm M.A. Clinical characteristics of chronic idiopathic intestinal pseudo-obstruction in adults. Gut. 41. 675-81 (1997)

84. Stanghellini V. et al. Natural history of intestinal failure induced by chronic idiopathic intestinal pseudo-obstruction. Transplant Proc. 42. 15-8 (2010)

85. Lindberg G., Iwarzon M. \& Tornblom H. Clinical features and long-term survival in chronic intestinal pseudo-obstruction and enteric dysmotility. Scand J Gastroenterol. 44. 692-9 (2009)

86. Mecoli C., Purohit S., Sandorfi N. \& Derk C.T. Mortality, recurrence, and hospital course of patients with systemic sclerosis-related acute intestinal pseudoobstruction. $J$ Rheumatol. 41. 2049-54 (2014)

87. Emmanuel A. V., Shand A. \& Kamm, M. A. Erythromycin for the treatment of chronic intestinal pseudo-obstruction: description of six cases with positive response.. Aliment Pharmacol Ther. 19. 687-694 (2004)

88. Emmanuel A.V. et al Randomised clinical trial: the efficacy of prucalopride in patients with chronic intestinal pseudo-obstruction--a double-blind, placebocontrolled, cross-over, multiple $\mathrm{n}=1$ study. Aliment Pharmacol Ther. 35. 48-55 (2012)

89. Ponec RJ, Saunders MD, Kimmey MB. Neostigmine for the treatment of acute colonic pseudo-obstruction. N Engl J Med. 341(3). 137-41 (1999)

90. Nikou G.C. et al. Effect of octreotide on intestinal motility and bacterial overgrowth in scleroderma. $N$ Engl J Med. 325(21). 1461-7 (1991)

91. Perlemuter G. et al. Octreotide treatment of chronic intestinal pseudoobstruction secondary to connective tissue diseases. Arthritis Rheum. 42. 1545-9 (1999)

92. Charalambopoulos D. and Sfikakis P.P. Treatment of small intestinal disease in systemic sclerosis with octreotide: a prospective study in seven patients. J Clin Rheumatol : Pract Rep Rheum Musculoskelet Dis. 13. 119-23 (2007)

93. Klein-Weigel P., Opitz C. \& Riemekasten G. Systemic sclerosis - a systematic overview: part 1-disease characteristics and classification, pathophysiologic concepts, and recommendations for diagnosis and surveillance. Vasa. 40. 6-19 (2011)

94. Ruiter G. et al. Iron deficiency in systemic sclerosis patients with and without pulmonary hypertension. Rheumatology (Oxford). 53. 285-92 (2014)

95. Ghrénassia E. et al. Prevalence, correlates and outcomes of gastric antral vascular ectasia in systemic sclerosis: a EUSTAR case-control study. J Rheumatol. 41. 99-105 (2014)

96. Marie I. et al. Watermelon stomach in systemic sclerosis: its incidence and management. Aliment Pharmacol Ther. 28. 412-21 (2008)

97. Ingraham K.M. et al. Gastric antral vascular ectasia in systemic sclerosis: demographics and disease predictors. J Rheumatol. 37.603-7 (2010) 
98. Sellinger C.P. and Ang Y.S. Gastric antral vascular ectasia (GAVE): an update on clinical presentation, pathophysiology and treatment. Digestion. 77:131-7 (2008)

99. Gostout C.J. et al. Endoscopic laser therapy for watermelon stomach. Gastroenterology. 96. 1462-5 (1989)

100. Shibukawa G. et al. Gastric antral vascular ectasia (GAVE) associated with systemic sclerosis: relapse after endoscopic treatment by argon plasma coagulation. Intern Med. 46. 279-83 (2007)

101. Swanson E., Mahgoub A., MacDonald R. \& Shaukat A. Medical and endoscopic therapies for angiodysplasia and gastric antral vascular ectasia: a systematic review. Clin Gastroenterol Hepatol. 12. 571-82 (2014)

102. Wells C.D. et al. Treatment of gastric antral vascular ectasia (watermelon stomach) with endoscopic band ligation. Gastrointest Endosc. 68. 231-6 (2008)

103. Watson M. et al. Gastric antral vascular ectasia (watermelon stomach) in patients with systemic sclerosis. Arthritis Rheum. 39. 341-6 (1996)

104. Cho S. et al. Endoscopic cryotherapy for the management of gastric antral vascular ectasia. Gastrointest Endosc.68. 895-902 (2008)

105. Schulz S.W. et al. Improvement of severe systemic sclerosis-associated gastric antral vascular ectasia following immunosuppressive treatment with intravenous cyclophosphamide. J Rheumatol. 36. 1653-6 (2009)

106. Sebastian S., O'Morain C.A. \& Buckley M.J. Review article: current therapeutic options for gastric antral vascular ectasia. Aliment Pharmacol Ther. 18. 157-65 (2003)

107. van Laar J.M. et al. Autologous hematopoietic stem cell transplantation vs intravenous pulse cyclophosphamide in diffuse cutaneous systemic sclerosis: a randomized clinical trial. JAMA. 311(24). 2490-8 (2014)

108. Bhattacharyya A. et al. Autologous hematopoietic stem cell transplant for systemic sclerosis improves anemia from gastric antral vascular ectasia. $J$ Rheumatol. 42. 554-5 (2015)

109. Battle W.M. et al. Abnormal colonic motility in progressive systemic sclerosis. Ann Intern Med. 94. 749-52 (1981)

110. Sacher P., Buchmann P. \& Burger H. Stenosis of the large intestine complicating scleroderma and mimicking a sigmoid carcinoma. Dis Colon Rectum. 26. 347-8 (1983)

111. Wang S.J. et al. Colonic transit disorders in systemic sclerosis. Clin Rheumatol. 20. 251-4 (2001)

112. Whitehead W.E., Taitelbaum G., Wigley F.M. \& Schuster M.M. Rectosigmoid motility and myoelectric activity in progressive systemic sclerosis. Gastroenterology. 96 (2 Pt 1). 428-32 (1989)

113. Emmanuel A., Tack J., Quigley E. \& Talley N. Pharmacological management of constipation. Neurogastroenterol Motility. 21(Suppl 2). 41-54 (2009)

114. Butt S. and Emmanuel A. Systemic sclerosis and the gut. Expert Rev Gastroenterol Hepatol. 7. 331-9 (2013)

115. Folwaczny C. et al. Effects of various prokinetic drugs on gastrointestinal transit times in patients with progressive systemic scleroderma. Z Gastroenterol. 35. 905-12 (1997)

116. Lindsey I., Farmer C.R. \& Cunningham I.G. Subtotal colectomy and cecosigmoid anastomosis for colonic systemic sclerosis: report of a case and review of the literature. Dis Colon Rectum. 46. 1706-11 (2003)

117. Davis R.P., Hines J.R. \& Flinn W.R. Scleroderma of the colon with obstruction: report of a case. Dis Colon Rectum. 19. 256-9 (1976) 
118. Shafik A., Shafik A.A., El-Sibai O. \& Ahmed I. Colonic pacing: a therapeutic option for the treatment of constipation due to total colonic inertia. Arch Surg. 139. 775-9 (2004)

119. Thaha M.A. et al. Sacral nerve stimulation for faecal incontinence and constipation in adults. Cochrane Database Syst Rev. 24. 8:CD004464 (2015)

120. Balbir-Gurman A., Brook O.R., Chermesh I. \& Braun-Moscovici Y. Pneumatosis cystoides intestinalis in scleroderma-related conditions. Intern Med J. 42. 323-9 (2012)

121. Andréasson K. et al. Faecal levels of calprotectin in systemic sclerosis are stable over time and are higher compared to primary Sjögren's syndrome and rheumatoid arthritis. Arthritis Res Ther. 16. R46 (2014)

122. Pazzi P. et al. Bile acid malabsorption in progressive systemic sclerosis. Gut. 29. 552-3 (1988)

123. Muir J.G. and Gibson P.R. The Low FODMAP Diet for Treatment of Irritable Bowel Syndrome and Other Gastrointestinal Disorders. Gastroenterol Hepatol ( $N$ Y). 9. 450-45 (2013)

124. Franck-Larsson K., Graf W., Ronnblom A. Lower gastrointestinal symptoms and quality of life in patients with systemic sclerosis: a population-based study. Eur $J$ Gastroenterol Hepatol. 21. 176-82 (2009)

125. Clements PJ, Becvar R, Drosos AA, et al. Assessment of gastrointestinal involvement. Clin Exp Rheumatol. 21 (3 Suppl. 29). S15-8 (2003)

126. Mawdsley A.H. Patient perception of UK scleroderma services-results of an anonymous questionnaire. Rheumatology. 45. 1573 (2006)

127. Thoua N. et al. Internal anal sphincter atrophy in patients with Systemic Sclerosis. Rheumatology. 50. 1596-1602 (2011)

128. Fynne L. et al. Distensibility of the anal canal in patients with systemic sclerosis: a study with the functional lumen imaging probe. Colorectal Dis. 15. e40-7 (2013)

129. Thoua NM, Abdel-Halim M, Forbes A, et al. Fecal Incontinence in Systemic Sclerosis Is Secondary to Neuropathy. Am J Gastroenterol. 107. 597-603 (2011)

130. Chiou A.W., Lin J.K. and Wang F.M. Anorectal abnormalities in progressive systemic sclerosis. Dis Colon Rectum. 32. 417-21 (1989)

131. Leighton J.A. et al. Anorectal dysfunction and rectal prolapse in progressive systemic sclerosis. Dis Colon Rectum. 36. 182-5 (1993)

132. Jaffin B.W., Chang P., Spiera H. Fecal incontinence in scleroderma. Clinical features, anorectal manometric findings, and their therapeutic implications. J Clin Gastroenterol; 25.513-7 (1997)

133. Denton C. and Black C. Scleroderma - clinical and pathological advances. Best Pract Res Clin Rheumatol. 18. 271-90 (2004)

134. Sporbeck B. et al. Effect of biofeedback and deep oscillation on Raynaud's phenomenon secondary to systemic sclerosis: results of a controlled prospective randomized clinical trial. Rheumatol Int. 32.1469-73 (2012)

135. Kenefick N.J. et al. Sacral nerve stimulation for faecal incontinence due to systemic sclerosis. Gut. 51. 881-3 (2002)

136. Butt $\mathrm{S}$. et al. Lack of effect of sacral nerve stimulation for incontinence in patients with systemic sclerosis. Colorectal Dis [Epub ahead of print] (2015)

137. Butt S.K. et al. Preliminary significant findings from a randomised controlled trial of posterior tibial nerve stimulation in systemic sclerosis associated faecal incontinence. UEG Journal. 2 (1 Suppl 1). A407 (2014) 
138. Bae S. et al. Associations between a scleroderma specific gastrointestinal instrument and objective tests of upper gastrointestinal involvements in systemic sclerosis. Clin Exp Rheumatol. 31 (2 Suppl 76). 57-63 (2013)

139. Alrubaiy L. et al. Systematic review of health-related quality of life measures for inflammatory bowel disease. J Crohns Colitis. 9. 284-92 (2015)

140. Spiegel B.M. et al. Development of the NIH Patient-Reported Outcomes Measurement Information System (PROMIS) gastrointestinal symptom scales. Am J Gastroenterol. 109. 1804-14 (2014)

141. Nagaraja V. et al. Construct validity of the Patient-Reported Outcomes Measurement Information System gastrointestinal symptom scales in systemic sclerosis. Arthritis Care Res (Hoboken). 66.1725-30 (2014)

Figure 1 | Small-intestinal involvement in systemic sclerosis is associated with marked physiological changes compared with normal tissue. [Au:Figure title OK? I've suggested text but please feel free to change as you see fit - note that the figure and legend must be understandable in isolation from the main text] The four key pathophysiological mechanisms causing gastrointestinal dysfunction in patients with systemic sclerosis are: infiltration of immune cells into gut smooth muscle; fibrosis of gut smooth muscle; labile vascular tone of the submucosal arterioles and venules; and enteric nervous system and smooth muscle dysfunction. ENS, enteric nervous system.

Figure 2 | Gastrointestinal presentations in systemic sclerosis according to anatomical location.

Figure 3 | Proposed pathway for screening and managing malnutrition in patients with systemic sclerosis. These recommendations are in the opinion of the author. [Au:OK?] CIPO, chronic intestinal pseudo-obstruction; GI, gastrointestinal; MDT, multidisciplinary team; MUST, malnutrition universal screening tool; SIBO, small intestinal bacterial overgrowth.

Table $1 \mid$ Potential differential diagnoses according to symptom presentation [Au:OK?]

\begin{tabular}{|l|l|l|}
\hline Symptom & Differential diagnosis & Assessment \\
\hline Dysphagia & Oesophageal dysmotility & Upper gastrointestinal \\
& Oesophageal candidiasis & endoscopy \\
& Peptic stricture & Oesophageal manometry \\
& Comorbid malignancy & $\begin{array}{l}\text { Ambulatory pH and } \\
\text { impedance }\end{array}$ \\
\hline
\end{tabular}




\begin{tabular}{|c|c|c|}
\hline Heartburn and regurgitation & $\begin{array}{l}\text { GERD } \\
\text { Barrett oesophagus } \\
\text { Oesophageal candidiasis }\end{array}$ & $\begin{array}{l}\text { Upper gastrointestinal } \\
\text { endoscopy } \\
\text { Ambulatory pH and } \\
\text { impedance }\end{array}$ \\
\hline Nausea and vomiting & $\begin{array}{l}\text { GERD } \\
\text { Gastroparesis } \\
\text { SIBO }\end{array}$ & $\begin{array}{l}\text { Upper gastrointestinal } \\
\text { endoscopy } \\
\text { Gastric scintigraphy } \\
\text { Hydrogen breath test }\end{array}$ \\
\hline Abdominal pain and distension & $\begin{array}{l}\text { Gastroparesis } \\
\text { SIBO } \\
\text { CIPO } \\
\text { Constipation }\end{array}$ & $\begin{array}{l}\text { Gastric scintigraphy } \\
\text { Hydrogen breath test } \\
\text { Abdominal X-ray or CT / } \\
\text { [Au:Do you mean X-ray } \\
\text { OR CT or CT or MRI in } \\
\text { this instance? Or do you } \\
\text { mean CT and/or MRI? } \\
\text { Or CT with MRI? Can } \\
\text { you please clarify for } \\
\text { other instances in the } \\
\text { table.] MRI of abdomen }\end{array}$ \\
\hline Weight loss & $\begin{array}{l}\text { Systemic comorbidity } \\
\text { (sepsis, drug related) } \\
\text { Reduced oral intake } \\
\text { (GERD and gastroparesis) } \\
\text { CIPO }\end{array}$ & $\begin{array}{l}\text { Blood screen } \\
\text { Careful history taking } \\
\text { Abdominal radiography or } \\
\text { CT/MRI of abdomen }\end{array}$ \\
\hline Constipation & $\begin{array}{l}\text { Slow-transit constipation } \\
\text { Puborectal dyssynergia } \\
\text { CIPO }\end{array}$ & $\begin{array}{l}\text { Transit study } \\
\text { MR proctogram } \\
\text { Abdominal X-ray or CT / } \\
\text { MRI of abdomen }\end{array}$ \\
\hline Diarrhoea & $\begin{array}{l}\text { Overflow diarrhoea } \\
\text { SIBO } \\
\text { Bile acid malabsorption } \\
\text { Coeliac disease } \\
\text { Fructose intolerance } \\
\text { Amyloidosis }\end{array}$ & $\begin{array}{l}\text { Digital rectal examination } \\
\text { Abdominal X-ray or CT / } \\
\text { MRI of abdomen } \\
\text { SeHCAT study } \\
\text { Serology while on gluten } \\
\text { Breath test } \\
\text { Lower gastrointestinal } \\
\text { endocopy and biopsy }\end{array}$ \\
\hline Faecal incontinence & $\begin{array}{l}\text { Systemic-sclerosis- } \\
\text { associated sphincter } \\
\text { atrophy } \\
\text { Comorbid obstetric injury } \\
\text { Comorbid diarrhoea }\end{array}$ & $\begin{array}{l}\text { Endoanal ultrasonography } \\
\text { and anorectal physiology }\end{array}$ \\
\hline
\end{tabular}

CIPO, chronic intestinal pseudo-obstruction; SeHCAT, selenium homocholic acid taurine; SIBO, small-intestinal bowel overgrowth 


\section{Acknowledgements:}

[Au:This section is optional, but you may use it to thank any funding bodies or acknowledge help with preparing the manuscript.]

The author is supported by the National Institute of Health Research UCLH Biomedical Research Centre.

\section{Competing interests}

The author declares no competing interests.

\section{Biography}

Anton Emmanuel is a neurogastroenterologist with a clinical and academic interest in gut

dysfunction in neurological and systemic diseases. Based at University College London, UK, he is fortunate to work with the tertiary systemic sclerosis service at the Royal Free Hospital, UK. Through this collaboration he has jointly supervised postgraduate research projects that have generated data contributing to this Review, and he has benefited from seeing first-hand the range of gastrointestinal presentations in patients with systemic sclerosis.

\section{Blurb:}

[Au: We include a brief ( 40 words) description of the Review in the Table of Contents, blurb OK? Please feel free to amend as you see fit]

Gastrointestinal dysfunction [Au:OK?] is very common in patients with systemic sclerosis, and often severely reduces quality of life. In this Review, Emmanuel discusses optimal strategies to identify and manage gastrointestinal complications in patients with systemic sclerosis, and outlines potential new therapies. 